

\section{HARVARD UNIVERSITY.}

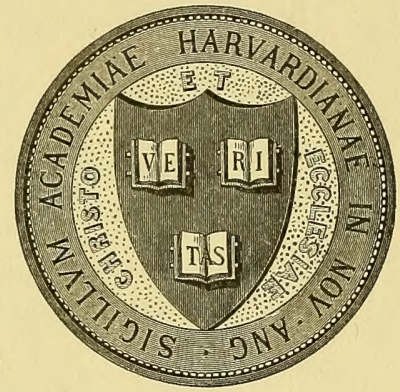

\section{LIBRARY}

OF THE

MUSEUM OF COMPARATIVE ZOÖLOGY 41,776

BEquest of

WILLIAM MCM. WOODWORTH.

\section{Decenter 29,1914.}




\section{1,776}

Ein weiterer Beitrag zur Statistik des

\section{Echinococcus hominis} in Pommern.

\section{Inaugural-Dissertation zur}

\section{Erlangung der Doctorwürde} in der

Mediein, Chirurgie und Geburtshülfe, welche nebst beigefügten Thesen

mit Zustimmung der Hohen Medicinischen Facultät der Königl. Universität Greifswald am Mittwoeh, den 4. Juli 1894 mittags 12 Uhr öffentlich verteidigen wird Richard Lehmann aus Fraustadt (Schiesien).

\section{Opponenten:}

Herr cand. med. Wied emann. Herr cand. med. Otto Müller.

\section{Greifswald.}

Druck von Julius Abel. 1894 . 


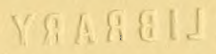

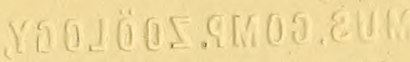

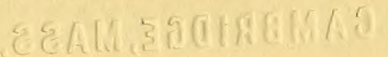


Seinen teuren Eltern 


\title{
in Liebe und Dankbarkeit
}

\author{
gewidmet
}


Wiederholt ist von Mosler, Hueter, v. Bardeleben, Vogt u. A. auf die Häufigkeit des Vorkommens der Echinococcen-Erkrankung in Neuvorpommern hingewiesen worden. Auf Veranlassung des Herrn Prof. Dr. Peiper hat Bahr im vorigen Jahre die in den Greifswalder klinischen Instituten beobachteten, sowie auch die aus den Sectionsprotokollen des pathologischen Instituts gezogenen Fälle in seiner Inaugural-Dissertation zusammengestellt. Ein grosser Teil der von Peiper und Bahr gesammelten Fälle stammt aus Neuvorpommern, die übrigen aus den andern Teilen dieser Provinz, bez. aus den benachbarten Provinzen.

Es lag der Gedanke nahe, eine solche Statistik, wie sie für Neuvorpommern angefertigt worden ist, auch für die übrigen Teile Pommerns aufzustellen, um so mehr, als doch alle diese Gebiete in natürlichem Zusammenhange unter einander stehen und auch die Beschäftigung und Lebensgewohnheiten der Bewohner - Ackerbau und Viehzucht - in ihnen die gleichen sind; und es ist doch unzweife!haft von Interesse, zu untersuchen, ob die Echinococcen-Erkrankung in dem übrigen Pommern in derselben Ausdehnung vorkommt, als in Neuvorpommern, dem nächst Mecklenburg ,klassischen“ Lande für diese Erkrankung in Deutschland. Sehr gern folgte ich daher einer liebenswürdigen Anregung des Herrn Prof. Dr. Peiper, eine solche Statistik zu bearbeiten.

Vor allen Dingen schien es mir interessant, das in den grossen Stettiner Krankenhäusern vorhandene Material 
kennen zu lernen. In liebenswürdiger Bereitwilligkeit haben die Herren: Dr. Sch uchardt, Direktor des städtischen Krankenhauses, Dr. Hans Sch midt, Direk tor des Krankenhauses Bethanien, die Krankenjournale mir zu diesem Zweck zur Verfügung gestellt. Es sei mir gestattet, auch an dieser Stelle den genannten Herren für ihr liebenswürdiges Entgegenkommen meinen ergebensten Dank auszusprechen. Sodann habe ich mich an eine grössere Anzahl von prakt. Ärzten der Provinz gewandt, die mir ebenfalls, soweit ihnen diesbezügliche Fälle vorgekommen sind, ihr Material überlassen haben, wofür ich auch ihnen meinen herzlichsten Dank sage. Es waren dies die Herren: Dr. Stein brück-Züllchow (2 Fälle); Dr. Schmidt-Polzin (2 Fälle); Dr. Kor tü mı-Swinemünde(2 Fälle); Dr. Schi n keUsedom (1 Fall); Dr. Schroeder-Pasewalk (1 Fall); Kr.Phys. Dr. Voigt-Cammin (1 Fall); Dr. Gerke-Cammin (1 Fall); Dr. Lebrom-Koeslin (2 Fälle); Dr. Buchterkirch-Stolp (1 Fall); Dr. Bohltz-Stargard (2 Fälle); Dr. Streuber-Stargard (1 Fall).

Schliesslich hat mir Herr Prof. Dr. Peiper noch einige Fälle zur Verfügung gestellt, die ihm ebenfalls von prakt. Ärzten der Provinz zugegangen waren, nämlich von den Herren: Dr. Knecht-Ückermünde (1 Fall); San.-Rat Dr. Schultze-Swinemünde (1 Fall); Dr. WaldowTreptow a./T. (1 Fall); Dr. Schlüter-Bredow (2 Fälle); Dr. Pritsch-Pölitz (2 Fälle); Dr. Hartwig-Pyritz (2 Fälle). Endlich habe ich diejenigen Fälle der Bahr'schen Statistik, die aus Alt-Vorpommern stammen, ebenfalls kurz angeführt resp. auf sie verwiesen, wobei ich natürlich auf eine genauere Beschreibung derselben verzichtet habe.

Was die äussere Anordnung des Materials betrifft, so ist dieselbe in ähnlicher Weise, wie in der Bahr'schen Dissertation erfolgt, d. h. die verschiedenen Fálle von 
Echinococcus-Krankheiten sind nach den befallenen Organen zusammengestellt und geordnet. Am Schluss erfolgt dann auf Grund des vorhandenen Materials eine Übersicht der Verbreitung der Erkrankung in den einzelnen Kreisen, soweit es möglich geworden ist, die Herkunft der Patienten $\mathrm{zu}$ ermitteln.

\section{Leberechinococcen.}

Fall I.

Aus dem Krankenhans Bethanien-Stettin. Beobachtet im Jahre 1881.

1. Frau Anna Habermann, Gastwirtsfrau, Kronheide bei Greifenhagen. Echinococcus hepatis. Geheilt durch Laparotomie.

\section{Fall II.}

Bethanien-Stettin. 1882.

2. Marie Dittmann, Kratznik, Kr. Randow. Echinococcus hepatis.

\section{Fall III.}

Bethanien-Stettin. 1883.

3. Gustav Wittnebel, Knecht aus Cantreck, Kr. Cammin. Echinococcus hepatis. Exitus letalis. Sektionsbefund: Echinococeus hepatis, perforirt nach der rechten Lunge. Eine Anzahl der Blasen war am Darm adhaerent.

Fall IV.

Bethanien-Stettin. 1884.

4. Ferdinand Erdmann, Maurermeister aus Treptow a. R., Kr. Greifenberg. Echinococcus hepatis. Operation. Exitus letalis. Sektion hat nicht stattgefunden.

$$
\text { Fall V. }
$$

Bethanien-Stettin. 1885.

5. Frau Emilie Engel, Herkunft nicht bekannt. Echinococcus hepatis. Bei der Operation fand sich eine über mannskopfgrosse Blase im rechten Leberlappen, durch welche die Leber vollständig nach links gedrängt war. 
Fall VI.

Bethanien-Stettin. 1885.

6. Max Splittgerber, Kind, Herkunft nicht bekannt. Echinococcus hepatis, von der unteren Seite des rechten Leberlappens ausgehend. Laparotomie. Exitus letalis.

Fall VII.

Bethanien-Stettin. 1886.

7. Ada Güstrow, Herkunit nicht bekannt. Echinococcus hepatis. Ungeheilt entlassen.

Fall VIII.

Bethanien-Stettin. 1887.

8. Frau Auguste Bluhm, Schiffersfrau, Gr. Ziegenort, Kr. Ueckermünde. Erkrankte mit Magen- und Verdauungs-Beschwerden. Die Magengegend erschien etwas vorgewölbt. Die Beschwerden bestanden seit einem Jahr. Echinococcus hepatis, an der unteren Fläche der Leber sitzend. Operation. Heilung. Alter der P. 34 Jahre.

\section{Fall IX.}

Bethanien-Stettin. 1887.

9. Gustar Lindenberg, Müller in Züllchow, Kr. Randow. 50 J. alt. Echinococcus hepatis. Operation. Heilung. Fall X.

Bethanien-Stettin. 1887.

10. Hermann Kersten, Arbeiter in Guttberg, Kr. Pyritz, 26 J. alt. Echinococcus hepatis. Exitus letalis.

Fall XI.

Bethanien-Stettin. 1888.

11. Max Clauss, Uhrmacher, Stettin. Echinococcus hepatis. Operation. Heilung.

\section{Fall XII.}

Mitgeteilt von Dr. Schinke-Usedom. 1888.

12. Minna Schultz, $2 \cdot \mathrm{J}$. alt, wohnhaft zu Usedom; beschäftigt im Haushalt und mit ländlichen Arbeiten, Tochter eines Viehhändlers. Echinococcus hepatis. Symp- 
tome: Schmerzeu in der Lebergegend beim Athmen; es traten Fiebererscheinungen auf, die mit normalen Temperaturen wechselten. Der Tumor liess sich palpieren; die Punktion desselben ergab eine himbeergeléeartige, mit Eiter gemischte Masse. Es handelte sich um einen vereiterten Leberechinococcus. Operation wurde abgelehnt. Exitus nach dreimonatlichem Krankenlager.

Fall XIII.

Mitgeteilt von Dr. Pritzch-Pölitz. 1888.

13. Schiffer Georg Budey, Jasenitz Kr. Randow; 51 J. alt. Kommt zur Behandlung mit Schmerzen in der Magengegend und der Leber. Leber geschwollen, erscheint vergrössert und schmerzhaft, Magengrube ebenso. Später zeigen sich im Abdomen zahlreiche kleinere Tumoren von Wallnuss- bis Hühnereigrösse. Operation in BethanienStettin resp. bei Dr. Schlüter-Bredow. Gestorben im Alter von 53 Jahren.

\section{Fall XIV.}

Mitgeteilt von Kr.-Phys. Dr. Voigt-Cammin.

14. Arbeiterfrau H. aus Brissenthin, Kr. Cammin; 38 J. alt. Echinococcus hepatis, vom linken Lappen ausgehend. Bei der Operation, durch welche Heilung erfolgte, fanden sich 75 Tochterblasen.

\section{Fall XV.}

Mitgeteilt von Dr. Gerke-Cammin.

Beobachtet i. J. 1885/86.

15. Frau Fieischfresser, $38 \mathrm{~J}$. alt, Fischhändlersfrau. Tumor des linken Leberlappens; später bildete sich in der Lebergegend eine Fistel, aus der sich serös-eitrige Flüssigkeit und Echinococcen-Blasen entleerten. Therapie: Erweiterung des Ganges, Drainage, Ausspülung. Nach 6 Monaten Heilung. I)ie Genesene ist bis heute gesund. 
Fall XVI.

Mitgeteilt von Dr. Lebram-Koeslin.

16. In mittleren Jahren stehender Gutsbesitzer aus der Koesliner Gegend. Beobachtet vor ca. 12 Jahren. Echinococcus hepatis. Operation im Augusta-Hospital in Berlin. Später erkranikte P. an Diabetes mellitus; ob letztere Affektion in irgend welchem ursächlichen $\mathrm{Zu}-$ sammenhang mit der ersteren stand, liess sich nicht entscheiden.

\section{Fall XVII.}

Mitgeteilt von Dr. Buchterkirch-Stolp;

beobachtet von dem verstorbenen Dr. Bumke vor ca. 15 Jahren.

17. Dame vom Lande. Echinococcus hepatis; Durchbruch nach dem rechten Pleuraraum. Operation, Heilung. Fall XVIII.

Mitgeteilt von Dr. Schroeder-Pasewalk. 1891.

18. Frau eines Schneidermeisters, 55 J. alt, Pasewalk. $\mathrm{Kr}$. Ückermünde. Echinococcus hepatis. Infection war wahrscheinlich von einem Schosshunde ausgegangen, der von der Patientin sehr verbätschelt wurde. Operation 1891 in der Greifswalder chirurgischen Klinik. Heilung. P. ist 2. Z. noch vollständig gesund.

Fall XIX.

Mitgeteilt von Dr. Streuber-Stargard.

Beobachtet i. J. 1868.

19. Frau Kaufmann Burg, Stargard, Kr. Saatzig. Echinococcus hepatis. Entleerung des Sackes durch Incision. Exitus letalis kurze Zeit nach diesem Eingriff. Fall XX.

Aus dem Sektionsprotokoll des städtischen Krankenhauses

Stettin. Dr. Schuchardt.

20. Betrifft einen alten decrepiden, moribund in das Krankenhaus eingelieferten Mann, bei welchem durch die 
Sektion ein vereiterter Leberechinococcus, der intra vitam keine Symptome gemacht hatte, als Nebenbefund festgestellt wurde.

\section{Fall XXI.}

Aus dem Sektionsprotokoll des städtischen Krankenhauses Stettin, Dr. Schuchardt.

21. Emma Guse, Mädchen. Bei der Sektion wurde ein kirschkerngrosser Leberechinococcus als Nebenbefund festgestellt.

\section{Fall XXII.}

Mitgeteilt von Dr. Steinbrück, dirigirendem Arzt des Johanniter-Krankenhauses in Züllchow bei Stettin.

22. In mittleren Jahren stehender Arbeiter aus Züllchow, Kr. Randow. Bemerkte eine rasch wachsende Geschwulst in der Lebergegend: Echinococcus hepatis. Operation. Heilung. Beobachtet Mitte der 80 er Jahre.

Fall XXIII.

Mitgeteilt von Dr. Knecht-Ǘckermünde, 1893.

Irrenheilanstalt.

23. August Neumann aus Züllchow, Kr. Randow. Wurde unter den Zeichen starker Abmagerung, sowie körperlich und geistig fortschreitender Paralyse in das Krankenbaus zu Ückermünde aufgenommen. Tod 4 Monate nach der Aufnahme. Bei der Sektion ergiebt sich ausser dem typischen Befund der Paralyse folgendes: in der Mitte des unteren Randes des Leberlappens sitzt eine von dem Lebergewebe deutlich abgegrenzte, faustgrosse Geschwulst, die sich schlaff fluktuirend anfühlt. Beim Einschneiden erwies sie sich als eine von einer dicken bindegewebigen Kapsel umbüllte glattwandige Höhle, aus der sich ein dicker, weissgelber, rahmiger Inhalt entleert, der zahlreiche erbsen- bis apfelgrosse, von einer durchscheinenden Bindegewebshülle umgebene Blasen enthält. 
Fall XXIV.

Chirurg. Klinik. Greifswald. 1894.

24. Rudolf Müller, $62 \mathrm{~J}$., Tischler, aus Camminke, Kr. Usedom-Wollin, Echinococcus hepatis. Heilung durch Operation.

\section{Fall XXV.}

Sektions-Protokoll des patholog. Instituts zu Greilswald. 189\%. No, 104.

25. Frau Wilke, $45 \mathrm{~J}$. alt, Müllersfrau aus Friedensfelde, Kr. Kammin. Klinische Diagnose: Bauchfellentzündung. Der rechte Leberlappen wölbt sich stark buckelförmig hervor. Bei der Palpation zeigt sich deutliche Fluktuation. Bei näherer Untersuchung erweist sich der Tumor als Echinococcus.

\section{Fall XXVI.}

Mitgeteilt von Dr. s'chlüter-Bredow. Beobachtet 1893.

26. Junge Frau, $28 \mathrm{~J}$. alt, aus Stettin stammend. Echinococcus des linken Leberlappens. Die Diagnose durch Probepunktion festgestellt. Operation wurde $a b-$ gelehnt; weiterer Verlauf nicht bekannt.

\section{Fall XXV1I.}

Aus der Greifswalder gynaekolog. Klinik. 1893.

27. Frau Baumann, 33 J. alt, aus Nossendorf, Kr. Demmin. P. hatte bereits vor 3 Jahren wegen heftiger Stiche in der rechten Seite einen Arzt consultirt, der damals schon das Vorhandensein eines Tumors festgestellt hatte. Bei der Untersuchung findet man in der rechten Seite einen bis zur Mittellinie reichenden, bis auf die Darmbeinschaufel ragenden, solide sich anfühlenden, auf der Oberfläche glatten Tumor, der nach aufwärts bis an den rechten Rippenbogen reicht. Ein fester, $12 \mathrm{~cm}$ breiter Strang verbindet unzweifelhaft den Tumor mit der Leber, Die Geschwulst ist nach rechts und links leicht ver- 
schieblich. Zweizeitige Operation. Heilung innerhalb 4 Wochen. Die Diagnose auf Echinoccocus wurde durch die Operation bestätigt.

Fall XXVIII.

Bahr, Inaug.-Dissertat. Fall No. 3.

28. Wilhelmine F., 17 J., aus Jarmen. Echinococcus hepatis.

\section{Fall XXIX.}

Bahr, Inaug..-Dissert. No. 38.

29. Minna W., 9 J. alt, aus Jarmen. Subphrenischer Echinococcus.

\section{Fall XXX.}

Bahr, Inaug.-Dissert. No. 8.

30. T., Arbeiter, 38 J. alt, aus Demmin. Echinococcus des linken Leberlappens.

Fall XXXI.

Bahr, Inaug.-Dissert. No. 17.

31. Emil Pr., 17 J. alt, Fleischerlehrling aus Demmin. Fall XXXII.

Bahr, Inaug.-Dissert. No. 31.

32. Frau Str., 32 J. alt, aus Schmarsow, Kr. Randow, Frau eines Schäfers. Echinococcus hepatis.

Fall XXXIII.

Bahr, Inaug.-Dissert. No. 80.

33. Anna W., Dienstmädchen, 21 J. alt, Grosshammer, Kr. Randow. Echinococcus hepatis, durch die Sektion festgestellt.

\section{Fall XXXIV.}

Bahr, Inaug.-Dissert. No. 13.

34 Dorothea P., 54 J. alt, Schäfersfrau aus Mellentin, Kr. Usedom-Wollin, Echinococcus hepatis.

Fall $\mathrm{XXXV}$.

Bahr, Inaug.-Dissert. No. 22.

35. Ludwig W., Zieglermeister, 37 J. alt, aus Ueckermünde. Echinococcus hepatis. 
Fall XXXVI.

Bahr, Inaug.-Dissert. No. 79.

36. Alwine P., 34 J. alt, Ueckermünde. Bei der Section ein wallnussgrosser Leberechinococcus.

\section{Fall XXXVII.}

Bahr, Jnaug.-Dissert. No. 77.

37. Bernhard K., 16 J. alt aus Eggesin, Kr. Ueckermünde. Bei der Sektion ergiebt sich ein Leberechinococcus, vom r. Lappen ausgehend.

\section{Fall XXXVIII.}

Bahr, Inaug.-Dissert. No. 25.

38. Christian R., 25 J. alt, Zimmermann aus Liebgarten, Kr. Ueckermünde. Echinococcus hepatis.

\section{Fall XXXIX.}

Mitgeteilt von Dr. Paul Hartwig-Pyritz. 1893.

39. Anna Heidemann, 22 J. alt, Köchin, erkrankte voriges Jahr an "Magenkrampf", von dem sie sich jedoch nach einiger Zeit wieder erholte. Im März dieses Jahres erkrankte sie aufs neue: es traten heftige Stiche im rechten Hypochondrium ein, Druckempfindlichkeit der Leber, hohes Fieber $\left(40^{\circ}\right)$, bedeutend vermehrte Pulsfrequenz (148-160 Schläge), starke Diarrhoen. Die Schmerzen zogen sich allmählich höher in der rechten Axillarlinie hinauf, ohne dass die Untersuchung der Lunge besondere Anhaltspunkte ergab. 14 Tage nach Beginn der Erkrankung zeigte sich rechts völlige Dämpfung, hochgradige Dyspnóe und vollständige Aphonie; das Abdomen schwoll und wurde bretthart und druckempfindlich. Die Diarrhoen wurden profuser. Starkes Oedem der Beine. 2 Tage vor dem Tode Erbrechen zahlreicher grösserer und kleinerer Echinococcusblasen.

Die Diagnose war zuerst gestellt worden auf Typhus abdominalis mit Perforationsperitonitis und Pleuritis exsu- 
dativa. Durch den Abgang der Blasen war jedoch die Diagnose Echinococcus sichergestellt. Wahrscheinlich hat es sich gehandelt um Echinococeus hepatis mit Durchbruch in die Lungen und den Darm. Eine Sektion hat nicht stattgefunden.

\section{Lungenechinococeen.}

Unter dieser Rubrik habe ich nur diejenigen Fälle zusammengestellt, die auf Grund der klinischen Diagnose als primär von der Lunge ausgehend angesehen worden sind, nicht aber auch diejenigen, bei denen die Lunge erst sekundär durch Übergreifen des Echinococcus von der Leber aus afficirt worden ist.

\section{Fall I.}

Mitgeteilt von Dr. Bohltz-Stargard.

40. Betrifft einen jetzigen Arzt, der zur Zeit seiner Erkrankung noch Schüler war; es handelte sich um Echinococeus pulmonum mit Durchbruch in die Bronchi. Es erfolgte Heilung.

\section{Fall II.}

Mitgeteilt von Dr. Kortüm-Swinemünde. Beobachtet i. J. 1886.

41. Ottilie B., Schlächtermeistersfrau und Tochter eines Schlächtermeisters in Swinemünde, $\mathrm{Kr}$. UsedomWollin, seit 14 Jahren verheiratet. Erkrankte vor 12 Jahren an Haemoptöe, die sich in kurzen Zwischenräumen wiederholte, bald abundant, bald in geringerem Maasse. Dieser Zustand dauerte 4 Jahre, wobei geringes Fieber zeitweise bestand. 1886 trat plötzlich höheres Fieber ein; eines Tages plötzlich starker Husten und Erstickungsnot, darauf Expectoration einer faustgrossen Blase mit Echinococcen. In den nächsten Tagen folgten noch zwei etwas kleinere Blasen. Das Krankenlager dauerte 3 Monate, 
worauf vollständige Heilung eintrat. Die Lunge ist etwas eingezogen. Auch in der Leber wird Echinococcus vermutet.

\section{Fall III.}

Mitgeteilt von Dr. Kortü m-Swinemünde.

42. Clara L., trat i. J. 1877 im Alter von 15 Jahren bei einem Schäfer in Dienst. In ihrem 28. Lebensjahre erkrankte sie an Lungenentzündung. L. H. U. Dämpfung. Allmählich begann die Expectoration, bis plötzlich grosse Mengen von Echinococcen-Blasen ausgehustet wurden. Diese Echinococeen-Expectoration dauerte 4 Wochen. Alsdann begann die Heilung, welche sich vollständig vollzog. Jetzt ist Patientin seit 3 Jahren verheiratet und Mutter mehrerer Kinder.

\section{Fall IV.}

Mitgeteilt von San. Rat Dr. Schultze-Swinemünde. Beobachtet i. J. 1881.

43. Amalie Lemke, 37 J. alt, geb. zu Stepenitz, Kr. Cammin. Eingeliefert in obiges Krankenhaus unter den Erscheinungen einer Bronchitis; dieselbe hatte schon längere Zeit gehüstelt und einige Tage lang war reichlich Auswurf eingetreten, welcher zahlreiche Echinococcen enthielt. Der Sitz der Erkrankung war der rechte untere Lungenlappen. Im Verlaufe einer Woche verschwanden die Echinococcen aus dem Auswurf, welch' letzerer nach einigen Tagen ganz aufhörte. Bei guter Pflege erholte sich Patientin wieder sehr rasch und konnte nach kurzer Zeit ihren Dienst wieder antreten.

Fall V.

Bahr, Inaug.-Dissert. Fall No. 101.

44. Hermann H., Ziegler aus Ückermünde, hustete eines Tages ohne Prodromalerscheinungen EchinococcenBlasen aus. Das Befinden wurde dadurch nicht alterirt. 
 \\ Echinococcen der Bauchhöhle. \\ Fall I. \\ Bahr, Inaug.-issertat. No. 113.}

45. Gustav Sch., aus Hohenmocker, Kr. Demmin, $14 \mathrm{~J}$. Rctorperitoneal liegender Tumor in abdomine. Durch die Operation erfolgte Heilung.

\section{Fall II.}

Bahr, Inaug.-Dissert. No. 114.

46. Frau Auguste Gr., ca. 35 J. alt, aus Demmin. Tumor in der rechten Lumbalgegend. Incision, Entleerung von Blasen. Exitus letalis 8 Monate nach der Operation.

\section{Fall III.}

Bahr, Inaug.-Dissert. No. 108.

47. Frau M., Gutspächterfrau aus Prienon, Kr. Demmin, $21 \mathrm{~J}$. alt. Tumor an der vorderen Scheidenwand, der bei der Geburt durch den Kopf des Kindes eingerissen wurde, wobei zahlreiche Blasen abgingen. Heilung.

\section{Fall IV.}

Bahr, Inaug.-Dissert. No. 106.

48. Johann B., 47 J. alt, Arbeiter ans Crummin, Kr. Usedom. Echinococcuscyste in der rechten Inguinalgegend. Operation. Heilung.

\section{Echinococcen der Milz.}

\section{Fall I.}

Bethanien - Stettin. 1886. Ergänzt von Dr. Waldow in Treptow a. T.

49. Albert Hagemeister zu Klentzow bei Treptow a. Toll., Schüler. Echinococcus lienis. Operation durch Dr. Maske-Stettin. Nach Verlauf von 4 Jahren entstanden bedeutende Schmerzen im Rücken links, sowie eine starke Anschwellung daselbst. Nach kurzer Zeit er- 
folgte Aufbruch mit Entleerung von viel Eiter und Blasen. Die Heilung dauerte 3 Monate. Seitdem kein Recidiv, Befinden sehr gut.

\section{Fall II.}

Mitgeteilt von Dr. Buddee-Wollin. 1893.

50. Luise Jarling aus Tonnin b. Wollin i. P.; Administratorstochter, $20 \mathrm{~J}$. alt. Echinococeus lienis. Operation in der Greifswalder chirurgischen Klinik. Heilung.

\section{Echinococeen der Niere. \\ Fall I.}

51. Mitgeteilt von Dr. Voigt-Anklam. Berichtet, dass er bei der Sektion eines aus dem Usedomer Kreise stammenden Mannes als Nebenbefund einen Nierenechinococcus gefunden habe.

\section{Fall II.}

Bethanien-Stettin 1892.

52. Frau Marie C., aus Ahlbeck, Kr. Usedom-Wollin 27 J. Echinococcus venis sinistri. Nephrotomie und Ausräumung des Sackes. Gebessert entlassen.

Echinococeen der Haut und Weichteile.

\section{Fall I.}

Mitgeteilt von Dr. Steinbrück-Züllchow.

Beobachtet Mitte der 80er Jahre.

53. Junger Mann von 18 Jahren, aus Züllchow, Kr. Randow. Bemerkte angeblich nach einem Stoss unterhalb des Kniegelenks eine rasch wachsende Geschwulst. Klinische Diagnose: Medulläres Sarkom. Vor der Operation wurde eine Probeincision in den Tumor gemacht, wobei sich letzterer als eine Echinococcus-Geschwulst der Weichteile entpuppt. Entleerung. Heilung. 


\section{Fall II.}

Mitgeteilt von Dr. Lebram-Koeslin.

Beobachtet ca. 1880.

54. Gutsbesitzer aus der Pollnower Gegend, Kr. Schlave. Echinococcus im Bindegewebe des Oberschenkels und Rückens. Operation. Heilung.

Fall III.

Mitgeteilt von Dr. Bohltz-Stargard.

Beobachtet ca. 1880.

55. Frau in mittleren Lebensjahren stehend, aus Stargard, Kr. Saatzig. Es bestand eine über zwei Fäuste grosse Geschwulst am Oberschenkel unmittelbar oberhalb des Knies. Bei der Operation stellte sich dieselbe als Echinococcus heraus. Die Heilung ging glatt vonstatten.

Fall IV.

Mitgeteilt von Dr. Paul Hartwig-Pyritz.

Beobachtet 1894.

Junges Mädchen, Anna Kaufmann, 29 J. alt, Plätterin. Geschwulst von Hühnereigrösse in der Kopf haut, die schon den Kopfknochen usurirt hatte. Klinische Diagnose: Atherom, das sich bei der Cperation, die von Dr. VierowStettin ausgeführt wurde, als Echinococeus entpuppte. Heilung.

\section{Echinococcen der Knochen.}

\section{Fall 1.}

Mitgeteilt von Dr. Schmidt-Polzin.

57. Wilhelmine Raddatz, geb. 1.9. 1859 zu Zühne, aufgenommen in das Polziner Krankenhaus im Dezember 1885, entlassen im Dezember 1886. Sie war nachweislich mit Hunden nicht in Berührung gekommen, wurde zu einer Badekur in das Polziner Krankenhaus eingeliefert, um wegen einer completen Lähmung beider Beine, der 
Blase una des Mastdarms, die auf ein rheumatisches Leiden zurückgeführt wurde, Heilung zu erlangen. Diese Lähmung hatte vor ca. 2 Jahren begonnen, ging unter der Behandlung fast ganz wieder zurück, um vor ca. 3 Monaten "iederzukehren und sich weiter auszubilden bis zu dem nachstehenden Befund. Bei der Untersuchung fand sich hinten auf der Wirbelsäule eine kleine, nussgrosse Erhöhung, die undeutlich fluctuirte. Bei einer Probepunktion ergiebt sich eine getrübte Flüssigkeit, in welcher sich bei mikroskopiseher Untersuchung Hackenkränze fanden, wonach die Diagnose auf Wirbelkörperechinococcus sichergestellt war. Eine Incision entleerte eine Menge Blasen; bei Erweiterung der Wunde gelangte man in eine apfelgrosse Höhle in den Körpern des elften und zwölften Brust- und ersten Lendenwirbels, die mit Blasen angefüllt war. Die Mutterblase wurde ebenfalls entfernt und konnte dabei eine knöcherne Abgrenzung des Rückenmarkkanals nach oben und unten festgestellt werden. Die Wunde wurde austamponirt, es erfolgte Heilung. Die Lähmungserscheinungen gingen jedoch trotz monatelanger Anwendung des constanten Stroms nicht zurück, nur die grossen Zehen konnten etwas bewegt werden bei der Entlassung aus dem Krankenhaus; nach wie vor musste jedoch Patientin katheterisirt werden. Sie lebte noch 4 Jahre.

\section{Fall II.}

Mitgeteilt von Dr. Sehmidt-Polzin.

58. Tagelöhner Albert Plantekow, geb. zu Neudorf, Kr. Bublitz, i. J. 1867. Aufgenommen im Juli 1887, gebeilt entlassen im September 1888. Kindskopfgrosser Echinococcus unterhalb der Nackenmuskulatur: ausgehend von der Wirbelsäule (sechster Halswirbel bis dritter Brustwirbel). Bei der Spaltung stellt es sich heraus, dass es nicht eine Mutterblase ist, sondern dass es sich um eine 
abgekapselte Abscesshöhle handelt, in der Eiter und kleine Echinococcenblasen schwimmen. Nach Entleerung der Höhle findet sich in derselben ein Gang, bei dessen Sondirung man ganz in der Tiefe auf Knochen kommt. Das Fieber, welches bestanden hatte, fiel ab, erhob sich jedoch nach ca. 5 Wochen wieder sehr hoch, sodass die Wunde wieder geöffnet und der Gang nach der Wirbelsäule hin ausgedehnt und drainirt wurde, aus dem dicken Drain entleeren sich öfter einige kleine Echinococcen-Blasen. Das Fieber fiel wiederum ab, um sich nach einigen Wochen bis über $40^{\circ} \mathrm{zu}$ erheben. Da die Dämpfung sich verstärkte und Husten sich einstellte, wurden die 3 oberen Pippen hinten oben resecirt und eine Verwachsung der Pleurablätter konstatiert. Sofort wurde wit dem Paquelin in die Lunge eingegangen, ohne jedoch auf den Herd zu gelangen. Die Wunde wird austamponiert. Am andern Morgen wurden plötzlich unter heftigen Erstickungserscheinungen mehrere Blasen mit Eiter und ein ca. bohnenstückgrosses Stück spongiösen Knochens durch Aushusten herausbefördert. Das Fieber hörte nunmehr definitiv anf, die Wunden schlossen sich gut. Patient vermietete sich einige Monate später als Pferdeknecht und konnte alle Arbeit verrichten.

\section{Fall III.}

Bethanien-Stettin. Dr. Hans Schmidt. 1892.

59. Fritz Mitzlaff, Gärtner, 25 J. alt, aus Augusthof, Kr. Pyritz. Echinococcus der ersten Rippe. Exstirpation. Heilung.

Zum Schluss füge ich hier einen Fall bei, der, was den Sitz des Echinococcus betrifft, jedenfalls zu den seltenen Vorkommnissen rechnet; es handelt sich nämlich um einen Echinococcus der Speicheldrüsen, mitgeteilt von Dr. Hans Schmidt, Bethanien-Stettin. 1893.

60. Emilie Retzlaff aus Dramburg. Echinococcus der 
glandula sublingualis sinistra. Die Drüse wurde in toto entfernt, erst dann aufgeschnitten, wobei sich in derselben ein nussgrosser Echinococcus fand.

Werfen wir noch einmal einen kurzen Blick auf die mitgeteilten Fälle, so ergiebt sich aus ihnen folgendes:

Die Gesamtzahl der mitgeteilten Fälle, einschliesslich der aus der Bah r'schen Dissertation mit hinübergenommenen beträgt 60 . Die einzelnen Organe waren in folgendem Verhältnis beteiligt:

Echinococcen der Leber . . . . 39 Fälle $=65,0 \%$ Echinococcen der Lungen . . . 5 Fälle $=8,3 \%$ Echinococcen der Haut und Weichteile 4 Fälle $=6,6^{\circ}$. Echinococcen der Milz . . . 2 Fälle $=3,3 \%$ Echinococcen der Niere . . . . 2 Fälle $=3,3 \%$ Echinococcen der Knochen .. . . 3 Fälle $=5,0 \%$ Echinococeen der Bauchhöhle . . 4 Fälle $=6,6 \%$

Den Fall von Echinococeus der Speicheldrüsen habe ich erst nicht mit in diese Organ-Statistik gezogen, weil er ja doch etwas extrem seltenes ist.

Auffallend könnte es erscheinen, dass die Echinococcen der freien Bauchhöhle sämtlich der Bahr'schen Statistik entstammen, während unter den von mir gesammelten kein einziger Fall zu verzeichnen ist.

Von den 60 Fällen gehörten an:

dem männlichen Geschlecht . . . . $27=45 \%$ dem weiblichen Geschlecht . . . . $33=55 \%$

Was das Alter der erkrankten Personen betrifft, so sind leider nur in einer beschränkten Anzahl von Fällen positive Angaben zu ermitteln gewesen, während bei den übrigen meist nur der Vermerk , in mittleren Jahren stehend" angegeben ist. A ber auch bei denjenigen Fällen, wo positive Altersangaben vorhanden sind, ist das mittlere 
Lebensalter vorherrschend, aus naheliegenden Gründen. $\mathrm{Tas}$ Kindesalter waren nur in 2 Fällen beteiligt. (No. 6, No. 29).

Wenn man bedenkt, wie lange Zeit unter Umständen Echinococcen im Körper sein können, ohne Symptome hervorzurufen, so wird man den Altersangaben inbezug auf die statistische Seite wenig Wert beilegen.

Was den Beruf der Erkraukten betrifit, so waren unter den männlichen Personen vertreten:

Gutsbesitzer, Schüler, Müller, Gärtner, Schiffer, Tagelöhner, Arbeiter, Schlächter; auffallend könnte es erscheinen, dass Schäfer, Hirten, Förster, Jäger so gut wie gar nicht vertreten sind. Von den weiblichen Personen wollen wir in dieser Beziehung nur hervorheben die Frau eines Schlächtermeisters und die Tochter eines Viehhändlers, sowie die Frau eines Schäfers.

Intra vitam wurden von den 60 mitgeteilten Fällen beobachtet 52, während in 8 Fällen Echinococcus erst als Nebenbefund bei der Sektion festgestellt worden ist.

Soweit sich die Herkunft der Patienten ermitteln liess, waren die einzelnen Kreise der Provinz, soweit sie in unserer Statistik in Betracht kommen, in folgendem Verhältnis beteiligt:

Greifenhagen: No. 1.

Kammin: No. 3, 14, 25.

Greifenberg: No. 4.

Saatzig: 19, 55.

Pyritz. No. 10, 39, 56, 59.

Bublitz 58.

Randow: No. 2, 9, 13, 22, 23, 32, 33, 53.

Ückermünde: No. 8, 18, 35, 36, 37, 38, 44.

Stettin: No. 11, 26.

Usedom-Wollin: No. 12, 24, 34, 41, 气0, 5๊1, 52.

Hinter-

pommern.

Demmin: No. 27, 28, 29, 30, 31.

Vor-

pommern. 
Ich bin nun weit entfernt davon zu behaupten, dass die von mir gebrachte Statistik wirklich alle beobachteten Fälle in sich schliesst; denn jeder, welcher mit den einschlägigen Verhältnissen vertraut ist, weiss, wie schwierig es für den Einzelnen ist, eine Statistik anzulertigen, die wirklich in jeder Beziehung Anspruch auf Vollständigkeit machen könnte. Aber selbst, wenn es mir möglich gewesen wäre, wirklich alle beobachteten Fälle zu sammeln, so würde auch dadurch eine absolute Vollständigkeit und ein abschliessendes Urteil über die Häufigkeit der Echinococcen-Erkrankung nicht erreicht sein; denn bekanntlich giebt es Echinococcen, die wegen ihrer geringen Grösse intra vitam völlig symptomlos verlaufen und erst dureh die Sektion als Nebenbefunde konstatiert werden. Sektionen werden jedoch in der Regel nur in grösseren Krankenhäusern resp. in pathologischen Instituten vorgenommen; wenn dagegen dem Privatpraxis ausübenden Arzt Fälle letztgenannter Art begegnen, so werden sie naturgemäss stets seiner Beobachtung sich entziehen.

Aber selbst wenn man alle diese Möglichkeiten in Betracht zieht, wird man doch zugeben müssen, dass die Zahl der mitgeteilten Fälle bedeutend zurückbleibt hinter den Zahlen, welche für Mecklenburg und Neuvorpommern in dieser Beziehung konstatiert worden sind, und dass somit aus der Statistik die Thatsache herrorgeht, dass die Echinococen-Erkrankung in Alt-Vor-und Hinterpommern viel seltener ist als in Neuvorpommern und Mecklenburg. Es ist doch auffallend, wenn z. B. in dem grossen städtischen Ḱrankenhause zu Stettin trotz des grossen Krankenmaterials kein einziger Fall zu eruieren war, der mit der Diagnose Echinoccceus autgenommen oder operativ behandelt worden wäre.

Das relativ grösste Material lieferte das Krankenhaus Bethanien-Stettin, wo mir die Krankenjournale der Jahr- 
gänge 1880-1893 zur Verfügung standen; aber auch hier steht die Zahl der beobachteten Fälle - 16 - in keinem Verhältnis zu der Zahl der während dieses Zeitraumes zur Behandlung gekommenen Patienten: 2079ã. Auch die Mitteilungen einer ganzen Reihe von praktischer Ärzten aus der Provinz erhärten die Thatsache, dass die Erkrankung in dem übrigen Pommern eine seltene ist im Vergleich zu Neuvorpommern.

So schreibt Dr. Schmidt-Polzin, dass „im Laufe seiner 10 jährigen Thätigkeit am hiesigen Krankenhause, in welches so ziemlich alle schwereren Fälle, die zwischen Stettin und Danzig vorkommen, eingeliefert werden, nur 2 Fälle von Echinococcus benbachtet worden sind; die Krankheit dürfte demnach wohl zu den seltenen grezäblt werden.

Dr. Buchterkirch-Stolp sagt, dass „die Erkrankung in Hinterpommern eine so seltene ist, dass ihm in 12 jähriger Praxis kein Fall vorgekommen ist, trotzdem ihm der grösste Teil aller zu operierenden Fälle ins Krankenhaus gebracht wird."

Kr.-Phys. Dr. Alexander-Belgard bemerkt: dass inm "während seiner 16 jährigen praktischen Thätigkeit Fälle von Echinococcus nicht vorgekommen sind.

So könnte ich noch eine ganze Reihe von Vakatanzeigen mitteilen, aus denen hervorgeht, dass von einem endemischen Vorkommen der Echinococcen-Erkrankung, wie es für Neuvorpommern festgestellt worden ist, in dem übrigen Pommern keine Rede ist, sondern dass hier vielmehr die Erkrankung zu den seltenen zu rechnen ist.

Es drängt sich deshalb die Frage auf, ob in Alt-Vorund Hinterpommern Momente vorhanden sind, welche eine solche Häufigkeit der Echinococcen-Krankheit, wie sie für Veuvorpommern und Mecklenburg besteht, nicht autkommen lassen. Es würde sich hierbei um die Untersuchung folgender Punkte handeln: 
1. Leiden die Hunde Alt-Vor- and Hinterpommerns weniger an Taenia Echinococcus als diejenigen von Mecklenburg und Nenvorpommern? Diese Frage zu beantworten bin ich ebenso ausserstande als Madelung, der die Frage aufwirft, ob etwa die Hunde Mecklenburgs mehr an Taenia Echinococeus leiden als anderwärts, denn die Vornahme von Hundesektionen gehört zu den grössten Ausnahmen und wird wohl nur in den seltensten Fällen ohne zwingenden Grund ausgeführt. Sehr wohl dagegen liesse sich diese Frage beantworten, wenn jeder verendete Hund tierärztlich zur Sektion gelangte und auf Taenia Echinococcus hin untersucht würde, wobei besonders auf diejenigen Hunde zu achten sein würde, welche, wie es ja auf dem Lande meist geschieht, bei den Schlachtungen der Haustiere zugegen sind. Allerdings ist die Ausführung dieser Massregel leichter gesagt als gethan; eine strenge Durchführung derselben wäre nur dann möglich, wenn eine a mtliche Anweisung bestände, wonach jeder Hundebesitzer verpflichtet ist, jeden in seinern Bereich verendeten Hund zur tierärztlichen Sektion zu bringen. Ob jedoch an den massgebenden Stellen Neigung zum Erlass einer derartigen Verordnung bestehen würde, möchte ich aus mehr als einem Grunde bezweifeln. Bei den jetzigen Verhältnissen ist jedenfalls eine Beantwortung obiger Frage unmöglieh.

2. Ist die Zahl der Hunde in Alt-Vor- und Hinterpommern eine wesentlich geringere als in Neuvorpommern und Mecklenburg? Soweit ich imstande war, darüber Erhebungen anzustellen, scheint das in der That der Fall zu sein. So z. B. beträgt die Zah] der Hunde in Stettin, das eine Einwohnerzahl von rund 125000 aufweist, 1628, d. h. 1 Hund kommt auf 70,6 Einwohner. Vergleichen wir damit den Hundebestand von einigen Städten Mecklenburgs, so kommt in Rostock 1 Hund auf 40,6 Einwohner, in Parchim auf 59,2 Einwohner, in Ludwigslust anf 18,5 
Einwohner. Offenbar hängt die niedrige Hundezahl in Stettin zusammen mit der hohen Hundesteuer, die dort besteht.

Ferner bin ich in der Lage, von einigen Gutsbezirken des Kreises Pyritz das Verhältnis der Einwohnerzahl zur Hundezahl mitzuteilen; die betreffenden Daten sind mir in liebenswürdiger Weise durch die Herren Rittergutsbesitzer: von Saldern, von Wedel, Nehring, von Schöning und Maywald mitgeteilt worden. Danach liegen die Verhältnisse so, dass auf den betreffenden Gütern resp. Gutsbezirken auf 1588 Einwohner 160 Hunde kommen, d. h. ungefähr auf 10 Einwohner 1 Hund. Wie es mit der Zahl der gehaltenen Hunde auf den ländlichen Gebieten Neuvorpommerns steht, darüber bin ich nicht in der Lage, irgend welche Angaben machen zu können.

3. Sind die Gelegenheiten zur Ansteckung in Alt-Vorund Hinterpommern vielleicht nicht so häufig als in Neuvorpommern und Mecklenburg? Madeluag, macht darauf aufmerksam, dass in Mecklenburg besonders früher, Hundekot als Hausmittel gegen verschiedenartige Krankheiten gebraucht wurde, und man könnte, da meines Wissens dieser Brauch in Alt-Vor- und Hinterpommern nich t besteht, denken, dass dieser Umstand etwas zur Erklärung beitragen möchte; wenn man jedoch bedenkt - worauf auch Madelung aufmerksam macht - dass nur alter Hundekot, in dem die Taenienkeime längst vernichtet sind, zu dem erwähnten Zweck angew endet wurde, so ist sofort klar, dass dieser Brauch nicht zur Erklärung herbeigezogen werden kann, weder nach der einen noch nach der andern Seite: weder kann das Vorhandensein dieses Brauches in Meoklenburg die Häufigkeit der Echinococcen-Erkrankung daselbst erklären noch das Nichtvorhandensein desselben die Seltenheit der Erkrankung in Alt-Vor- und Hinterpommern.

Auch wird man kaum annehmen dürfen, dass etwa 
in Alt-Vor- und Hinterpommern der persönliche Verkehr des Menschen mit dem Hunde nicht so intim ist wie in Necklenburg und Neuvorpommern; ich möchte bezweifeln, dass hierin ein wesentlicher ['nterschied zwischen beiden Gebieten besteht.

4. Wie steht es mit dem Bestand an Schlachtvieh in Alt-Vor- und Hinterpommern resp. mit der Verbreitung der Echinococcen-Krankheit beim Schlachtvieh. Diese Frage hat ein enorm praktisches Interesse und steht in sehr inniger Beziehung zu der Frage über die Häufigkeit der Echinococeen-Erkrankung beim Menschen. Denn II a delung hat speciell für Mecklenburg nachgewiesen, dass die Häufigkeit der Echinocoecen-Erkrankung abhängig ist von der Zahl der gehaltenen Haustiere - Rinder, Schweine, Schafe - , und dass gerade die Zahl der Schafe besonders hierbei ins Gewicht fällt; er hat nämlich gefunden, dass in denjenigen Teilen Mecklenburgs, wo der Echinoccocus beim Menschen am häufigsten vorkommt, nämlich im Norden und Osten, die Zahl der Schafe am grössten ist, während die Zahl der Rinder und Schweine in alien Teilen des Landes annähernd gleich ist; dass ferner dort, wo die Echinococcen-Krankheit beim Menschen am häufigsten vorkommt, dieselbe auch bei den Schafen am häufigsten beobachtet wird.

Es handelt sich also im vorliegenden Falle darum, zu untersuchen:

1. Ist der Bestandteil an Schlachtvieh, speziell an Schafen, in Alt-Vor- und Hinterpommern ein wesentlich geringerer als in Mecklenburg und Neuvorpommern?

2. Wie steht es mit der Verbreitung der EchinococcenKrankheit beim Schlachtvieh, speziell bei den Schaten?

Auf Grund der vom Kgl. statistischen Bureau herausgegebenen Statistik verhält sich der Bestand an Schlachtvieh in den einzelnen Kreisen folgendermassen: 


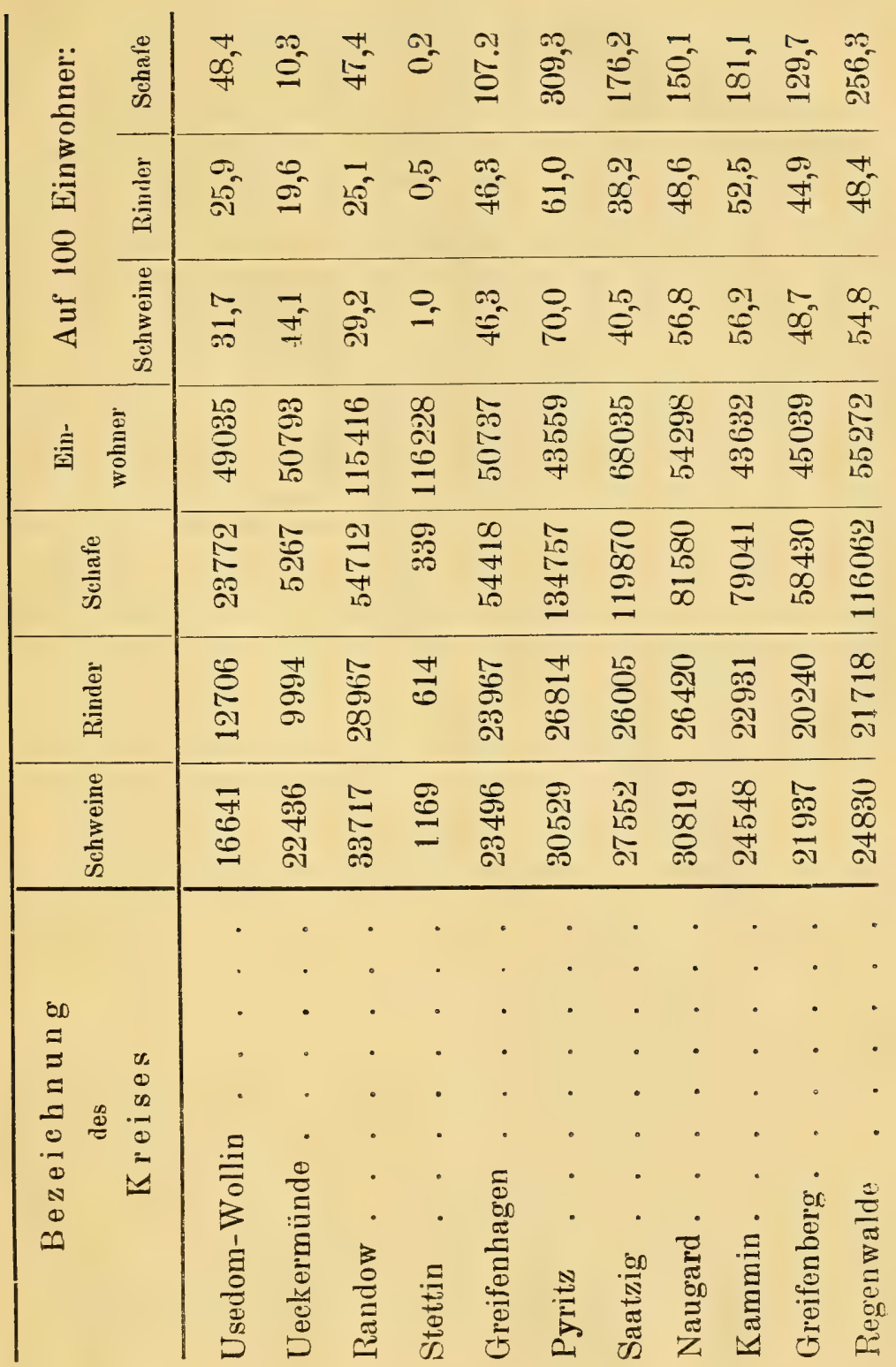


Aus dieser Tabolle geht hervor, dass die Kreise Pyritz und Regenwalde inbezug auf die Zahl der Schafe die übrigen Kreise weit übertreffen, ja dass sogar in dieser Beziehung ihnen Mecklenburg und Neuvorpommern nachstehen. Man müsste demnach vermuten, dass die Zahl der beobachteten Echinococcenfälle in diesen beiden Kreisen eine grosse sein möge, jedenfalls grösser als in den übrigen Kreisen, was jedoch, wie die oben angeführte, nach Kreisen geordnete Statistik zeigt, durchaus nicht zutrifft.

Eine Erklärung hierfür ist nur möglich, wenn die Echinococcen-Erkrankung beim Schlachtvieh, speciell bei den Schafen, in Alt-Vor- und Hinterpommern nicht so bäufig vorkommt, als in Neuvorpommern und Mecklenburg. Letzteres ist in der That der Fall. Wie die von Pientka aufgestellte Statistik über die Verbreitung der EchinococcenKrankheit beim Schlachtvieh ergiebt, wurden z. B.| in dem Schlachthause zu Stettin (Schlachth.-Dir. Falk) in der Zeit vom 1. Juni 1893 bis 30. November als echinococenhaltig befunden:

Rinder $7,10 \%$

Schweine $7,58 \%$

Schafe $19,13 \%$.

Im Schlachthaus zu Swinemünde in der Zeit vom 1. VII. bis 30. XI. 93 :

Rinder $25,00 \%$

Schweine $13,84 \%$

Schafe $20,50 \%$.

Im Sehlachthaus zu Koeslin in der Zeit vom I. VII. 93 bis 31. XII. 93:

Rinder $17,66 \%$

Schweine 0,73\%

Schafe $10,70 \%$.

Im Schlachthaus zu Stolp in der Zeit vom 1. VI. 93 bis 31. X. 93: 
Rinder 13,32\%

Schweine $2,77 \%$

Schafe $9,51 \%$.

In Regenwalde, wo kein Schlachthaus besteht, wo aber seit $1 \frac{1}{2}$ Jahren die obligatorische Fleischschau eingerichtet ist, wurden nach Mitteilungen des prakt. Tierarztes Herın Silber i. J. 1893 geschlachtet:

Schweine: 483 ; mit E. behaltet $4=0,9 \%$

Kühe: 193 ; mit E. behaftet $4=2 \%$

Schafe: 677 ; mit E. behaftet $15=2,2 \%$.

Vergleicht man damit die für Greifswald und Schwerin giltigen Zahlen, so wurden in Greifswald in der Zeit vom 1. VII. 93 bis 10. XII. 93 für echinocuccenhaltig befunden:

Rinder $64,58 \%$

Schweine $4,93 \%$

Schafe 51,02\%

In Schwerin in der Zeit vom 1. VI. 93 bis 10. XI. 93:

Rinder $30,15 \%$

Schweine $2,96 \%$

Schafe $42, \overline{0} 6 \%$

Es lässt sich somit auf Grun dieser Tabellen die Thatsache aufstellen, dass in Alt-Vor- und Hinterpommern die Echınococcen-Erkrankung peim Schlachtvieh, speziell bei den Schafen, seltener ist als in Mecklenburg und Neuvorpommern und dass daraus auch das seltene Vorkommen dieser Erkrankung beim Menschen in Alt- Tor- und Hinterpommern zu erklären sein dürfte, da ja ohne Zweifel der Hund unter gewöhnlichen Verhältnissen von den Schlachttieren die junge Echinococcen-Brut bezieht.

Auffallend ist allerdings, dass in Rostock die Zahl der Echinococcen-haltigen Schlachttiere eine äusserst geringe ist, obwohl doch Rostock von Madelung als derjenige Ort bezeichnet wird, in welchem bedeutend mehr Fälle von Echinococcus beim Menschen beobachtet sind, 
als überhaupt irgendwo im Grossherzogtum Mecklenburg. Für diese so widersprechende Thatsache sucht auch Pientka vergebens nach einer Erklärung; „sie müsste denn etwa, "sagt Pientka," darin liegen, dass zwischen Abfassung des Madelungschen Werkes und unserer (Pientikas) Statistik ein Zeitraum von über 10 Jahren liegt, in welcher Zeit in und um Rostock durch zweckmässige Massnahmen die Echinococcen-Krankheit bei Tieren auf die oben angegebenen Werte reduziert worden ist."

Jedenfalls geht aus allen diesen Momenten hervor, dass die Beantwortung der Frage, warum die EchinococenErkrankung in Gebieten, die geographisch einander nahe liegen und in denen auch die Lebensbedingungen im wesentlichen die gleichen sind, ein so überaus variables Vorkommen zeigt, noch manche Schwierigkeit zu überwinden hat.

Zum Schlusse erfülle ich die angenehme Pflicht, Herrn Prof. Dr. Peiper meinen herzlichsten Dank auszusprechen, der mich nicht nur mit seinem liebenswürdigen Rat bei der Ausführung der Arbeit unterstützt hat, sondern auch um die Beschaffung und Vervollständigung des Materials in hervorragender Weise bemüht gewesen ist. 


\section{Litteratur.}

Madelung: Beiträge mechlenburgischer Ärzte zur Lehre von der Echino. cocceri-Krankheit.

Bahr: Ein Beitrag zur Keuntuis der Echinococcen-Krankheit in Vorpommern. (Inaug.-Dissert. Greifswald 1893).

Pientka: Statistischer Beitrag zur Verbreitung der Echinococcen-Krankheit. (Inaug.-Dissert. Greifswald, 1894).

Kgl. preuss. statist. Burean: die vorläufgen Ergebnisse der Viehzähluug im preussischen Staat. 1892. 


\section{Lebenslauf.}

Richard Wilhelm Ulrich Lehmann, Sohn des Lazareth-Verwaltungs-Inspektors Friedrich Lehmann zu Schweidnitz i. Schles, evang., wurde am 8. Januar 1871 zu Fraustadt (Prov. Posen) geboren. Seine Vorbildung erhielt er auf dem Realgymnasium seiner Vaterstadt, später auf dem Gymnasium zu Gr.-Glogau und auf dem Elisabech-Gymnasium in Breslau, welches er Ostern 1890 mit dem Zeugnis der Reife verliess. Er bezog die Universität Breslau, um Jedizin zu studieren und bestand daselbst Ostern 1892 das Tentamen physicum. Michaelis 1892 verliess er Breslau, um seine Studien in Greifswald fortzusetzen. Hier bestand er am 19. Februar 1894 das Examen rigorosum.

Während seiner Studienzeit besuchte er die Vorlesungen, Kurse und Kliniken folgender Herren Professoren und Dozenten:

\section{In Breslau:}

Born, F. Cohn, H. Cohn, Cuhn, Filehne, E. Fränkel, C. Hasse, R. Heidenhain, Hürthle, Kast, Kaufmann, Ladenburg, O. E. Meyer.

\section{In Greifswald:}

Grawitz, L. Heidenhain, Helferich, Hoffmann, Krabler, Loeffler, Mosler, Peiper, Pernice, v. Preuschen, O. Schirmer, Schulz, Stoewer, Strübing.

Als Volontär durfte er thätig sein in den Kliniken der Professoren:

Neisser (Breslau), Geh. Rat Mosler, Geh. Rat Pernice.

Allen diesen seinen hochverehrten Herren Lehrern spricht Verfasser an dieser Stelle seinen herzlichsten Dank aus. 


\section{Thesen.}

I.

Die Injektion von indifferenten Lösungen in die Urethra bei einer floriden Gonorrhöe ist streng zu meiden.

II.

Die Exstirpation des syphilitischen Primäraffekts ist in allen Fällen, wo die Lokalisation es erlaubt und noch keine lange Zeit seit der Infektion verstrichen ist, auszuführen.

III.

Die Annahme, dass die croupöse Pneumonie im Kindesalter eine extrem seltene Erkrankung sei, ist nicht berechtigt. 



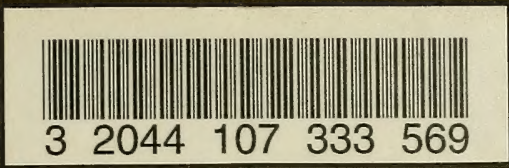


